\title{
Antiemetic Use in Acetaminophen Poisoning: How Does the Route of N-acetylcysteine Administration Affect Utilization?
}

\author{
Melissa A. Miller, PharmD ${ }^{a}$, Marisela Navarro, PharmD ${ }^{a}$, Steven B. Bird, MD $^{b}$, \\ Jennifer L. Donovan, PharmD ${ }^{a, b, c}$
}

aDepartment of Pharmacy, UMass Memorial Medical Center

bDepartment of Emergency Medicine University of Massachusetts Medical School

cDepartment of Pharmacy Practice Massachusetts College of Pharmacy and Health Sciences

\begin{abstract}
Introduction: We sought to compare antiemetic use after acetaminophen poisoning in patients treated with oral or intravenous (IV) N-acetylcysteine (NAC).

Methods: Our retrospective chart review identified 20 orally treated patients and 17 IV-treated patients. For both groups, we calculated the total number of antiemetic doses given, their associated cost, and also determined parameters that correlated with antiemetic use.

Results: IV-treated patients received fewer total antiemetic doses than those receiving oral NAC $(1.1 \pm 0.2 \mathrm{vs} .2 .8 \pm 0.7 ; \mathrm{P}=0.04)$. Antiemetic cost correlated with doses received for both groups; however, because the regression lines differed ( $\mathrm{P}=0.02)$, antiemetic therapy cost was less in IV-treated patients. In addition, serum acetaminophen concentration correlated with total antiemetic doses in oral NAC patients $(\mathrm{P}<0.002)$ but not with IV treatment patients $(\mathrm{P}=0.78)$.

Conclusions: Intravenous NAC reduced antiemetic utilization, and it costs less than oral therapy. Furthermore, antiemetic use appeared to be determined by a combination of acetaminophen concentration and NAC administration route.
\end{abstract}

\section{INTRODUCTION}

The most effective therapy for acetaminophen (APAP) poisoning is $\mathrm{N}$-acetylcysteine (NAC) [1]. Nevertheless, NAC's sulfur moiety gives it a "rotten egg" smell and taste and so its oral administration frequently exacerbates the nausea and vomiting associated with APAP poisoning itself $[2,3]$. Thus, repeated antiemetic doses are often needed to ensure adequate delivery of the antidote [4]. In 2004, the Food and Drug Administration approved an intravenous (IV) NAC preparation, Acetadote (Cumberland Pharmaceuticals Inc., Nashville, TN). Whether one route of NAC administration provides more effective therapy than another remains unknown; in fact, one open-label trial in pediatric patients found no difference between either oral or intravenous administration [5]. Thus, other factors may influence which NAC preparation is used; for example, the risk of adverse events and cost [6]. The IV product is significantly more expensive and hence some institutions limit the IV formulation to patients with fulminant hepatic failure, gastrointestinal bleeding or obstruction, or intolerance to oral NAC. An additional concern, antiemetic use, has received relatively little consideration as a factor in selecting NAC administration route, even though emesis has a major impact on patient comfort

Keywords: acetaminophen, antiemetics, n-acetylcysteine, overdose

Notes: There was no outside funding of any kind used for this study.

Corresponding Author: Jennifer L. Donovan, PharmD, Department of Pharmacy Practice, Massachusetts College of Pharmacy and Health Sciences, 19 Foster Street, Worcester, MA 01608. Email: Jennifer.Donovan@mcphs.edu 
as well as on the total treatment expense. Therefore, we had two goals:

(1) to compare antiemetic use and cost in patients receiving oral versus IV NAC for APAP poisoning, and

(2) to examine the determinants of antiemetic use for both delivery methods.

\section{MATERIALS AND METHODS}

We performed this Institutional Review Board approved study at a level one tertiary care university teaching hospital and regional toxicology referral center. We retrospectively evaluated the medical records of all patients, admitted between July 2002 and May 2004, who received oral NAC for a diagnosed APAP overdose. In our comparative group, we retrospectively identified patients receiving IV NAC (Acetadote) between July 2004 and September 2005. Both groups were identified using the hospital's databases. Each patient's medical chart was examined for demographic characteristics, admission and maximum serum APAP concentration, and the concurrent use of coingestants, including acute and chronic ethanol use. To assess the extent of liver injury and dysfunction, we recorded the peak values of the following parameters; alanine aminotransferase (ALT), aspartate aminotransferase (AST), total bilirubin, prothrombin time (PT), and International Normalized Ratio (INR). A full course of oral NAC or IV NAC was required for inclusion unless a toxicology service consultation recommended a shorter course of therapy:

1. Oral NAC equaled $140 \mathrm{mg} \mathrm{kg}^{-1}$ loading dose followed by $70 \mathrm{mg} \mathrm{kg}^{-1}$ every 4 hours for 17 doses, and

2. IV NAC equaled $150 \mathrm{mg} \mathrm{kg}^{-1}$ for 1 hour followed by $15 \mathrm{mg} \mathrm{kg}^{-1} \mathrm{hr}^{-1}$ for 4 hours followed by $7.5 \mathrm{mg} \mathrm{kg}^{-1} \mathrm{hr}^{-1}$ for 18 hours-dose based on an earlier study using IV NAC compounded from the oral preparation) [7].

Our hospital policy is to reserve IV NAC for those patients with fulminant hepatic failure, patient refusal, patient inability to tolerate oral NAC, when a nasogastric tube is not an option, or a gastrointestinal bleed or obstruction is present; otherwise, oral NAC is preferred. Patients were excluded from analysis if they were mechanically ventilated at any time, received a neuromuscular blocking agent, were pregnant, or were less than 12 years of age. Since our institution does not have a formal antiemetic administration protocol for APAP poisoning, we defined antiemetic utilization as ondansetron or metoclopramide (the two agents on our hospital's formulary) administered at any time after the start of NAC treatment. We considered a single dose as an utilization irrespective of the prescribed amount. The total number of antiemetic doses given to each patient was recorded. The average wholesale price, obtained from the RedBook, was used to calculate antiemetic treatment cost [8]. Finally, to examine potential determinants of antiemetic use, we sought to determine if the total number of antiemetic doses for oral and IV routes of NAC administration correlated with any of the other measured parameters, specifically serum APAP concentration and liver function tests.

\section{Statistical Analysis}

We used unpaired t-tests to gather group comparisons of continuous variables. If the data were normally distributed, but had different standard deviations, we used the Welch t-test. For data that were not normally distributed (assessed using the KolmogorovSmirnov test), we used the Mann-Whitney test. Non-continuous variables were analyzed using Fisher's Exact test. We performed linear regression analysis and used ANCOVA for subsequent statistical comparison of the regression lines. Odds ratio (OR) and relative risk (RR) were calculated using the approximation of Woolf and Katz, respectively (GraphPad InStat version 3.06). Data are presented as mean \pm standard error, and group differences were considered significant if $\mathrm{P}<0.05$.

\section{RESULTS}

We identified 54 oral NAC patients and 57 IV NAC patients, and we included 20 oral NAC patients and 17 IV NAC patients. As previously described, the most common reason for exclusion was failure to receive the full course of NAC. There were no differences between the oral and IV NAC groups with respect to gender, serum APAP concentration on arrival to the Emergency Department, time from ingestion to serum admission APAP concentration, maximum serum APAP concentration, concurrent coingestant, or ethanol utilization. However, oral NAC patients were younger than those in the IV group (Table 1). Most patients ingested other substances; the most commonly ingested was diphenhydramine or antihistamine agent, and this finding was similar between groups.

\section{Liver Injury and Dysfunction}

The measured injury/dysfunction parameters were all elevated above normal; however, there were no significant group differences (Table 2). Thus, our groups appeared well matched in terms of the severity of injury.

\section{Antiemetic Utilization and Cost}

A smaller percentage of IV-treated patients received any antiemetic therapy (categorized on a yes or no basis regardless of the total amount) than the oral group [58.8\% $(n=10)$ vs. $75.0 \%(n=15)]$; however, this difference was not significant [RR $0.7895 \%$ confidence interval $(0.48-1.25) ; \mathrm{P}=0.48]$. In contrast, IV-treated patients did receive fewer total antiemetic doses $(1.1 \pm 0.2$ vs. $2.8 \pm 0.7 ; \mathrm{P}=0.04$ ) (Figure 1). The greater use of ondansetron in the oral group was the major contributing difference $(1.5 \pm 0.5$ vs. $0.5 \pm 0.1$ doses; $\mathrm{P}=0.04$ ); there was no difference in metoclopramide use between groups $(\mathrm{P}=0.11)$. As expected, the greater antiemetic usage in patients receiving oral NAC was associated with greater cost; however, the large standard error prevented this $70 \%$ reduction from achieving statistical significance (oral: $\$ 49.31 \pm 19.64$ vs. IV: $\$ 14.70 \pm 4.92 ; \mathrm{P}=0.10)$. Nevertheless, as anticipated, we found highly significant linear correlations between antiemetic doses and cost for both groups (oral: $\mathrm{r}=0.8$; $\mathrm{P}<0.0001$ and IV: $\mathrm{r}=0.7 ; \mathrm{P}=0.0005)$. Furthermore, ANCOVA 
Table 1: Demographic and Clinical Data

\begin{tabular}{|c|c|c|c|c|}
\hline & $\begin{array}{l}\text { Oral NAC } \\
(\mathbf{n}=\mathbf{2 0})\end{array}$ & $\begin{array}{l}\text { IV NAC } \\
(n=17)\end{array}$ & $\begin{array}{l}\text { Odds Ratio } 95 \% \\
\text { Confidence Interval }\end{array}$ & P-Value \\
\hline Age (years) & $25.0 \pm 2.4$ & $37.2 \pm 4.8$ & NA & 0.03 \\
\hline Gender (\% female) & 80 & 77 & $0.8(0.1-3.8)$ & 1 \\
\hline \multicolumn{5}{|l|}{$\begin{array}{l}\text { Serum APAP concentration } \\
\left(\mu \mathrm{g} \mathrm{ml} \mathrm{m}^{-1}\right)\end{array}$} \\
\hline Admission & $107 \pm 22$ & $148 \pm 34$ & NA & 0.32 \\
\hline Maximum & $145 \pm 29$ & $156 \pm 34$ & NA & 0.81 \\
\hline $\begin{array}{l}\text { Time from reported ingestion } \\
\text { to serum admission APAP } \\
\text { concentration (hours) }\end{array}$ & $16.1 \pm 5.1$ & $28.8 \pm 9.3$ & NA & 0.26 \\
\hline Co-ingestants (\%) & 80 & 58.8 & $0.3(0.1-1.5)$ & 0.27 \\
\hline Acute ethanol (\%) & 25 & 17.6 & $0.6(0.1-3.2)$ & 0.7 \\
\hline Chronic ethanol (\%) & 30 & 23.5 & $0.7(0.1-3.1)$ & 0.72 \\
\hline \multicolumn{5}{|c|}{ APAP - acetaminophen; NAC - N-acetylcysteine } \\
\hline \multicolumn{5}{|c|}{ Data shown as mean \pm standard error of the mean; NA - not applicable } \\
\hline
\end{tabular}

\section{Table 2: Liver Function Data}

\begin{tabular}{lccc}
\hline & $\begin{array}{c}\text { Oral NAC } \\
(\mathbf{n}=\mathbf{2 0})\end{array}$ & $\begin{array}{c}\text { IV NAC } \\
(\mathbf{n}=\mathbf{1 7})\end{array}$ & P-Value \\
\hline Peak ALT $\left(\mathrm{IU} \mathrm{L}^{-1}\right)$ & $1,000 \pm 600$ & $1,000 \pm 700$ & 0.89 \\
\hline Peak AST $\left(\mathrm{IU} \mathrm{L} \mathrm{L}^{-1}\right)$ & $600 \pm 300$ & $1,200 \pm 900$ & 0.73 \\
\hline Total bilirubin $\left(\mathrm{mg} \mathrm{dL}^{-1}\right)$ & $1.5 \pm 0.2$ & $1.9 \pm 0.4$ & 0.38 \\
\hline $\begin{array}{l}\text { Prothrombin time }(\mathrm{s}) \\
\text { INR }\end{array}$ & $15.1 \pm 1.5$ & $16.2 \pm 2.3$ & 0.75 \\
\hline $\begin{array}{l}\text { ALT }=\text { alanine aminotransferase } \\
\text { AST }=\text { aspartate aminotransferase } \\
\text { INR }=\text { International Normalized Ratio } \\
\text { Data shown as mean } \pm \text { standard error of the mean. }\end{array}$ & & 0.67 \\
\hline
\end{tabular}

revealed a significant difference between the two regression lines $(\mathrm{P}=0.02)$, and so the gradient of the regression for the IV group was less than that for the oral group. Hence, the cost was less for any given number of antiemetic doses.

\section{Determinants of Antiemetic Use}

We found a significantly positive correlation between admission serum APAP concentration and the total number of antiemetic doses received in patients treated with oral NAC $(\mathrm{P}<0.002$; Figure 2). In contrast, there was no such relationship in IV-treated patients $(\mathrm{P}=0.78)$. Similar results were also obtained if the maximum serum APAP value was used (oral: $\mathrm{P}=0.02$; IV: $\mathrm{P}=0.91$, data not shown). There were no significant correlations between antiemetic doses and any of the liver injury/function parameters (data not shown).

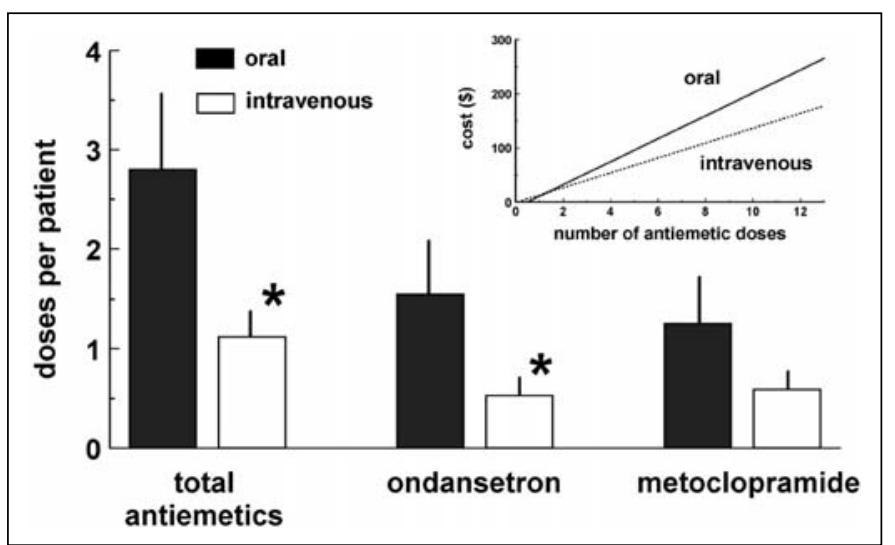

Figure 1: Intravenous (IV) N-acetylcysteine (NAC) treated patients required less total antiemetic doses and less ondansetron than the oral group. There was no difference in metoclopramide use. [* denotes $P<0.05]$

Inset-Antiemetic cost correlated with the number of antiemetic doses for both groups; however, for any given dose, the cost associated with IV NAC was lower $(P<0.02)$.

\section{DISCUSSION}

As far as we are aware, ours is the first specific evaluation of the use and determinants of antiemetic therapy in acetaminophen poisoning. We found that antiemetic use was less for patients who received IV NAC than for those who received oral NAC, a reduction attributed to IV-treated patients receiving less ondansetron. Although the cost savings attributed to antiemetic use in our study are insufficient to offset the greater expense associated with IV NAC administration, the potential patient benefit 


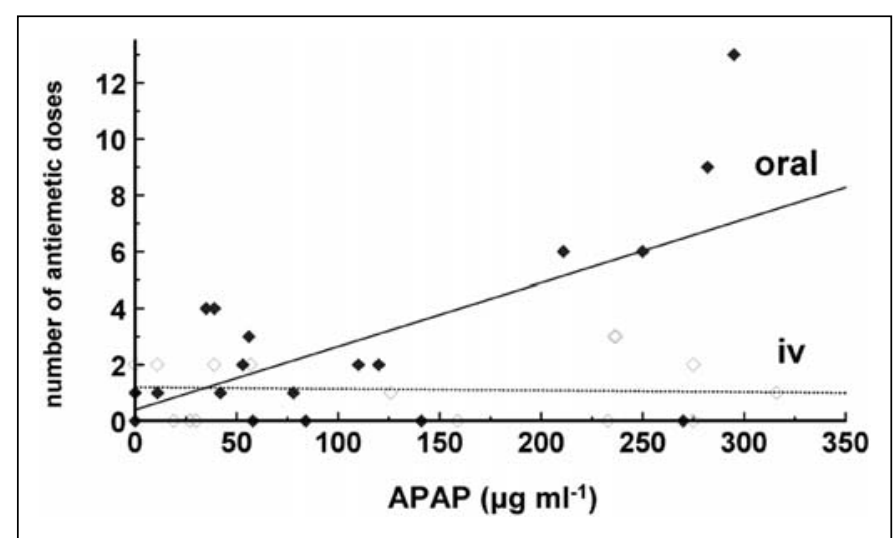

Figure 2: Antiemetic dose correlated with admission serum acetaminophen (APAP) concentration for patients receiving oral n-acetylcysteine (NAC)-black diamonds, but not for those treated with intravenous (IV) NAC-open diamonds

(such as less antiemetic need because of less nausea and vomiting) merits consideration. Nevertheless, a cost-effectiveness study would need to be performed to address these issues.

Evidence from the oncology and anesthesiology literature suggests that ondansetron is a more effective antiemetic than metoclopramide in preventing nausea and vomiting [9]. However, the results found in chemotherapy or anesthesiology literature may be inappropriate to extrapolate to treatment in APAP poisoning. A recent comparison of antiemetic therapy in an Emergency Department found that a $10 \mathrm{mg}$ IV dose of metoclopramide was no more effective than saline in diminishing patients' nausea (for any etiology, assessed using a visual analog scale) 30 minutes after treatment [10]. In addition, Wright and colleagues found that a 20-50 mg dose of metoclopramide significantly reduced emesis associated with oral NAC therapy for acetaminophen poisoning versus the standard dose of less than $20 \mathrm{mg}$ [11]. Thus, it is possible that patients in our study treated with oral NAC received more ondansetron; not because their symptoms were more pronounced, but because $10 \mathrm{mg}$ of metoclopramide had no effect. Similarly, the optimal dose of ondansetron for this indication is unknown. And our study is consistent with previous work that ondansetron may be needed to treat prior antiemetic failure [4].

Ondansetron is often reserved for either the most severe cases or those that fail to respond to other antiemetics $[4,12]$. These factors, together with the knowledge that oral NAC administration is itself associated with nausea and vomiting, initially prompted us to conclude that patients receiving oral NAC therapy appeared more severely affected by nausea and vomiting because of the route of NAC administration. We had initially excluded the possibility that serum APAP concentration contributed to the difference in antiemetic use because neither the admission nor the maximum value differed between groups; in fact, the admission value was slightly higher in the IV-treated patients (Table 1). Nevertheless, further examination revealed the possibility that APAP concentration and oral NAC administration contributed to increased antiemetic use (Figure 2). Thus, we speculate that antiemetic use might be determined by a combination of APAP concentration and the NAC administration route; however, the relative contribution of each remains unknown.

When performing comparisons of different treatment approaches, it is crucial to have well-matched groups. There was no statistical difference between the groups for serum APAP concentration, time from ingestion to admission of serum APAP concentration, the incidence of concurrent co-ingestants, nor in the severity of liver injury (Tables 1 and 2). Although comparison of mean enzyme levels can be misleading because of the data's nonGaussian distribution, we also found no group differences when we divided the data according to the criteria used by James and colleagues of mild $\left(<100 \mathrm{IU} \mathrm{L}^{-1}\right)$, moderate $(>100$, but $<1,000$ $\left.\mathrm{IU} \mathrm{L}^{-1}\right)$, and severe $\left(>1,000 \mathrm{IU} \mathrm{L}^{-1}\right)$ [13]. For all of the parameters measured, the only difference was that the IV-treated patients were older. It is possible that this age difference might also be associated with body mass differences and hence reduced antiemetic dose on a per kilogram basis in the IV group. If this were the case, the net effect could be expected to increase antiemetic use in the IV group, which was not found and so this statistical difference is unlikely to influence our findings.

\section{Limitations}

Because this was a retrospective study, our data collection was limited to information contained in the medical records. The time between acetaminophen ingestion and hospital arrival can influence antiemetic need; however, this information was only available for a limited number of the patients. Other factors, such as charcoal or cathartic agent administration or the prophylactic use of antiemetics, could also affect our results. Charcoal was given to 6 of the 37 patients (no difference between groups); however no incidences of cathartic agent or prophylactic antiemetic administration were recorded. In addition, our sample size was small and applicability to the greater population is limited. Also, the duration of NAC therapy may also influence emetogenicity. Although our data were gathered over two different time periods, the treatment protocols in practice at our hospital during this time were the same, as were the majority of the toxicologists. Hence, we do not think that the observed differences were merely a function of time.

\section{CONCLUSION}

The absence of direct evidence supporting the superior efficacy of one administration route instead of another suggests that other factors will influence the decision to treat acetaminophen poisoning with either oral or intravenous $\mathrm{N}$-acetylcysteine. We found that intravenous administration was associated with reduced antiemetic use (specifically less ondansetron) and a concomitant cost reduction suggests that this approach does have some distinct advantages. The correlation between the admission of acetaminophen serum concentration and antiemetic doses for oral but not intravenous treatment suggests that antiemetic use is determined 
by a combination of APAP concentration and the NAC administration route.

The authors have no potential financial conflicts of interest to report.

\section{REFERENCES}

1. Smilkstein MJ, Knapp GL, Kulig KW, Rumack BH. Efficacy of oral N-Acetylcysteine in the treatment of acetaminophen overdose. Analysis of the national multicenter study (1976 to 1985). N Engl J Med. 1988;319:1557-1562.

2. Vale JA, Proudfoot AT. Paracetamol (acetaminophen) poisoning. Lancet. 1995;346:547-552.

3. Linden $\mathrm{CH}$, Rumack BH. Acetaminophen overdose. Emerg Med Clin North Am. 1984;2:103-119.

4. Scharman EJ. Use of ondansetron and other antiemetics in the management of toxic acetaminophen ingestions. J Toxicol Clin Toxicol. 1998;36:19-25.

5. Perry HE, Shannon MW. Efficacy of oral versus intravenous $\mathrm{N}$-Acetylcysteine in acetaminophen overdose: Results of an open-label, clinical trial. J Pediatr. 1998;132:149-152.

6. Ternullo S. Acetadote (intravenous acetylcysteine): adverse effects more significant than with oral acetylcysteine. J Emerg Nurs. 2006;32:98-100.
7. Kao LW, Kirk MA, Furbee RB, Mehta NH, Skinner JR, Brizendine EJ. What is the rate of adverse events after oral $\mathrm{n}$-acetylcysteine administered by the intravenous route to patients with suspected acetaminophen poisoning? Ann Emerg Med. 2003;42:741-750.

8. Murray L. Red Book. Montvale, NJ: Thomson, 2005.

9. Kovac AL. Benefits and risks of newer treatments for chemotherapy-induced and postoperative nausea and vomiting. Drug Saf. 2003;26:227-259.

10. Braude D, Soliz T, Crandall C, Hendey G, Andrews J, Weichenthal L. Antiemetics in the ED: a randomized trial comparing 3 common agents. Am J Emerg Med.

2006; $24: 177-182$.

11. Wright RO, Anderson AC, Lesko SL, Woolf AD, Linakis JG, Lewander WJ. Effect of metoclopramide dose on preventing emesis after oral administration of N-Acetylcysteine for acetaminophen overdose. J Toxicol Clin Toxicol.

1999; $37: 35-42$.

12. Clark F, Chen R, Williams SR, Johnson CL, Harchelroad F. The use of ondansetron in the treatment of nausea and vomiting associated with acetaminophen poisoning. J Toxicol Clin Toxicol. 1996;34:163-167.

13. James LP, Simpson PM, Farrar HC, Kearns GL, Wasserman GS, Blumer JL, et al. Cytokines and toxicity in acetaminophen overdose. J Clin Pharmacol. 2005;45:1165-1171. 